Sir,

$\mathrm{Mr}$ Mayo is probably right when he says that MacNeill and I should not have included the pedigrees of McConville, West, and McAdams, but there is no certainty in such matters at present, because the current definition of Alport's syndrome may be too restrictive or too inclusive. In any case, the general thesis that one should not pool pedigrees with different characteristics is not persuasive. What MacNeill and I set out to do was to pool a number of pedigrees, admittedly somewhat different among themselves, to see whether abnormal ratios are observable in the totals, and whether they fit the explanation of Shaw and Glover. I cannot see the harm in pooling pedigrees for this purpose. In biological studies if we waited until our materials were free of unexplained variation, we would never be able to begin.

Mr Mayo has shown by statistical test that pedigrees for the syndrome are heterogeneous. I think that is true. The pedigrees analysed by Glover and myself showed differences in some of their ratios. I know of an unpublished pedigree in which deafness is absent, but the other manifestations, including abnormal segregation, are present. It does, indeed, appear that Alport's syndrome is quite variable, both among members of a family and among pedigrees. It has never seemed to me urgent to demonstrate this variability statistically, as I could not see that such a demonstration would show anything very illuminating. Probably pedigrees of all hereditary diseases are heterogeneous for reasons of genetic modification and environmental influences. Alport's syndrome is clearly more variable than many. Hence statistical proof of heterogeneity among the pedigrees does not constitute an advance in knowledge unless some pattern can be shown. Is the variation continuous? bimodal ? dichotonous? (Professor Clarke Fraser has suggested to me that there may be two kinds of Alport's syndrome with and without the abnormal ratios.) Are there correlations with climate or diet, or with ethnic origin? Are severity of symptoms and degree of abnormal segregation correlated? I am inclined to suppose that the cause of the disease is a chromosomal rearrangement and that in different families the break points are different or different linked modifiers are at work. But that is only speculation. Dietary effects, observed by Alport, remain to be followed up.

Mayo's finding of larger family size when a parent is affected strikes me as surprising and interesting. It is not entirely clear why he thinks it is an artefact, but in any event, it neither supports nor refutes anything MacNeill and I have claimed. We did not calculate any fitness values. The phrase about our interest in reproductive fitness which Mayo quotes is taken out of a sentence which says that one of our initial purposes was to investigate this subject. But in fact we abandoned that plan. The plan was mentioned in the paper by way of explaining why only pedigrees from the US were analysed.

Incidentally, I still think the Shaw and Glover explanation of this disease is by far the best available, and I regret now that MacNeill and I acquiesced at the instance of one of the assessors and toned down the conclusions given in the summary. The Shaw and Glover hypothesis is still in the running, but our summary appears to say that we favour no hypothesis. It seems to me better science to put forward strongly the best hypothesis available and see if other workers are stimulated to disprove it or to find a more appealing one. The alternative may be a rather dull and self-congratulatory consensus that nothing is known and nothing can be.

$$
\text { Yours, etc, }
$$

Richard F. Shaw

Department of Epidemiology,

University of Sherbrooke,

Quebec, Canada.

\section{The Crouzon Syndrome}

Sir,

We strongly object to the diagnosis of craniofacial dysostosis (Crouzon syndrome) in the two sibs reported recently by Juberg and Chambers (1973). Despite the shallow orbits present in both patients, the lack of proptosis rules out the Crouzon syndrome, which is characterized minimally by craniosynostosis, midface hypoplasia, and shallow orbits with secondary proptosis (Cohen, 1973). Occasionally, craniosynostosis may even be absent, as in several affected family members reportedo by Shiller (1959).

The Crouzon syndrome follows an autosomal domi nant mode of transmission with complete penetrance and variable expressivity. The possibility of genetic heterogeneity should always be kept in mind. However, that an autosomal recessive form of the Crouzon syndrome exists still remains to be shown.

The patients reported by Juberg and Chambers (1973) represent an isolated form of craniosynostosis, consistent with autosomal recessive inheritance. One of us (M.M.C.) has also observed several instances of affected sibs with isolated craniosynostosis.

Yours, etc,

Kenneth L. Jones and M. Michael Cohen, Jr.

Departments of Pediatrics and Orthodontics,

Schools of Medicine and Dentistry,

University of Washington,

Seattle, Washington, USA.

\section{REFERENCES}

Cohen, M. M., Jr. (1973). An etiologic and nosologic overview of craniosynostosis syndromes. Birth Defects: Original Article Series. (In press.)

Juberg, R. C. and Chambers, S. R. (1973). An autosomal recessive form of craniofacial dysostosis (the Crouzon syndrome). fournal of Medical Genetics, 10, 89-93.

Shiller, J. G. (1959). Craniofacial dysostosis of Crouzon: a case report and pedigree with emphasis on heredity. Pediatrics, 23, 107-112. 
Sir,

The usefulness of defining minimal diagnostic criteria for the Crouzon syndrome-for that matter, for any syndrome-depends upon both the quality and quantity of data research as well as on the arbitrary decisions of whoever establishes the criteria. To set up some criteria should not be difficult, but to do so to stand the test of time may be something else again.

Two terms, proptosis and exophthalmos, have often been used interchangeably to indicate abnormal protrusion of the eyeball. At least one authority (TrevorRoper, 1962) has suggested that exophthalmos should refer to forward protrusion of the eyeball alone; proptosis should indicate displacement of the lids and orbital contents as well as the eyeball. Most authors have used exophthalmos in their description of patients with craniofacial dysostosis (Hilson, 1947; Flippen, 1950; Pinkerton and Pinkerton, 1952; Dodge et al, 1959; Shiller, 1959; Vulliamy and Normandale, 1966). However, proptosis would seem to be the more accurate term because the whole content of the orbit would be expected to be abnormally projected as a result of orbital shallowness.

Although quantitative determination of the degree of protrusion has been possible by various designs of exophthalmometer for quite some time (the Hertel instrument dates from 1905), clinical judgement by standing behind the patient and looking over the forehead or by viewing from the side, has usually sufficed. Thus, most reports do not include quantitative assessment of the ocular abnormality but rather state only the result of clinical opinion. Consequently, some patients who by appearance have abnormal protrusion might by measurement and comparison be clearly beyond normal limits, but others might not. Normal values for 10 to 14 year olds have only recently been published (Gerber et al, 1972).

The exophthalmometric measurement of the female sib we reported (Juberg and Chambers, 1973) falls within the normal range for her age according to published data (Gerber et al, 1972), but we cannot classify her male sib because of the lack of comparative data. In our report we indicated the lack of abnormality of the position of the eyes of the two sibs judged by adult standards. However, the standards published are for Caucasians, and our patients are Negroes. R. C. Juberg, F. G. Sholte, Jr., and W. J. Touchstone (in preparation) have recently found by examination of 1219 Negroes, aged 5 to 11 years, that the means for both the internal intercanthal and the external intercanthal distances are greater than the normal values reported for Caucasians. If the width of the orbit as reflected by canthal measurements differs in racial groups, then it seems reasonable that the depth may also differ. Normal values, when they become available, will permit comparison of measurements in patients such as we reported.

$$
\text { Yours, etc, }
$$

Richard C. Juberg and Sue R. Chambers

Birth Defects Center, Department of Pediatrics, Louisiana State University School of Medicine in Shreveport,

PO Box 3932, Shreveport, Louisiana 71130, USA

\section{REFERENCES}

Dodge, H. W., Jr., Wood, M. W., and Kennedy, R. L. J. (1959). Craniofacial dysostosis : Crouzon's disease. Pediatrics, 23, 98-106.

Flippen, J. H., Jr. (1950). Cranio-facial dysostosis of Crouzon. Report of a case in which the malformation occurred in four generations. Pediatrics, 5, 90-96.

Gerber, F. R., Taylor, F. H., deLevie, M., Drash, A. L., and Kenny, F. M. (1972). Normal standards for exophthalmomentry in children 10 to 14 years of age: Relation to age, height, weight, and sexual maturation. Fournal of Pediatrics, 81, 327-329.

Hilson, D. (1947). Scaphocephaly in a brother and sister. An unusual incidence of this form of premature synostosis of the skull bones. Clinical Proceedings, 6, 64-70.

Juberg, R. C. and Chambers, S. R. (1973). An autosomal recessive form of craniofacial dysostosis (the Crouzon Syndrome). Fournal of Medical Genetics, 10, 89-94.

Pinkerton, O. D. and Pinkerton, F. J. (1952). Hereditary craniofacial dysplasia. American fournal of Ophthalmology, 35, 500-506.

Shiller, J. G. (1959). Craniofacial dysostosis of Crouzon. A case report and pedigree with emphasis on heredity. Pediatrics, 23, 107112.

Trevor-Roper, P. D. (1962). Ophthalmology: A Textbook for Diploma Students. Year Book, Chicago.

Vulliamy, D. G. and Normandale, P. A. (1966). Cranio-facial dysostosis in a Dorset family. Archives of Disease in Childhood, 41, 375-382. 